\title{
Rigid Gas permeable (RGP) contact lens induced microbial keratitis in a keratoconus patient: A case report.
}

\author{
Krishnendu Mandal. M.OPT ${ }^{1}$, Bhupesh Bagga. FRCS ${ }^{2}$, \\ Sobia Taureen. B. OPT ${ }^{3}$ \\ ${ }^{1,3}$ Optometry, L.V.Prasad Eye Institute, india \\ ${ }^{2}$ Ophthalmology, L.V.Prasad Eye Institute, india
}

\begin{abstract}
Introduction: To report a case of microbial keratitis in an individual with keratoconus using rigid gas permeable contact lenses.

Method: - A 23-year-old male presented with a history of pain, redness, photophobia, watering in right eye and his vision was reduced upon awakening. He was a known case of bilateral keratoconus who used rigid gas permeable contact lenses in both eyes on daily wear basis. Slitlamp examination revealed a paracentral stromal infiltrate in right eye. Corneal scrapings were collected for culture. Both contact lenses and lenses cleaning solution were collected for microbiological investigations.
\end{abstract}

Result: - Corneal scraping revealed plenty gram-negative bacteria (Pseudomonas aeruginosa) and contact lenses cleaning solution revealed klebsiella.

Conclusion:- Although microbial keratitis is an uncommon complication with rigid gas permeable contact lenses in keratoconus patients but it can be managed by proper microbiological work up and intensive medical care.

Key words:KC-Keratoconus, RGP- rigid gas permeable contact lens, MK-Microbial keratitis, Pseudomonas aeruginosa, Klebsiella.

\section{Introduction}

Keratoconus is a bilateral non-inflammatory progressive corneal thinning characterized by localized conical protrusion, irregular astigmatism and central scarring of the cornea., ${ }^{1,2}$ Keratoconus is usually well managed by soft or rigid gas permeable contact lenses where spectacles do not give satisfactory visual improvement. ${ }^{3}$ The most popular contact lenses are silicon hydrogel and rigid gas permeable contact lenses due to their high oxygen permeability. ${ }^{4}$ The complications are less prevalent with rigid gas permeable lenses as compare to soft contact lenses. ${ }^{5,6}$ The annual incidence of microbial keratitis in soft contact lenses wearer are more than that of rigid gas permeable contact lens users. ${ }^{5.6,7,8}$

Microbial keratitis is an uncommon complication of rigid gas permeable contact lenses associated with keratoconus. Although microbial keratitis has been well described in the literature as a complication with contact lenses, with rigid gas permeable contact lenses in keratoconus patient is described in the literature as an extremely rare event. ${ }^{5,6,7,9}$

We report a case of microbial keratitis in an individual with keratoconus who was using rigid gas permeable contact lenses.

\section{Case report: -}

A 23-year old male presented with pain, redness, photophobia, watering in right eye. He had been diagnosed with advanced keratoconus in both eyes four years prior. The patient had been using rigid gas permeable contact lens in right eye and Rose-k contact lens in left eye since three months. He denied any history of known ocular trauma, sleeping with contact lenses or any other systemic illness. He was wearing contact lenses overnight for approximately 16 hours.

Ophthalmic examination revealed an uncorrected visual acuity of 20/600 in right eye and 20/125 in left eye. Slitlamp examination of right eye revealed bulbar conjunctival congestion, cornea showing inferior paracentral anterior stromal infiltrate measuring in $2 \mathrm{~mm}$ horizontally and $1 \mathrm{~mm}$ in vertically associated with surrounding stromal oedema. Both eyes were having signs of advanced keratoconus. The patient was advised to discontinue contact lenses in both eyes. Diagnosis of Microbial keratitis made and corneal scraping was done. Both contact lenses and lens cleaning solution were collected for culture [Figure 1 and Figure 2]. Microbiological reports confirmed Pseudomonas Aeruginosa grown in the Chocolate agar culture medium and klebsiella in contact lens cleaning solution. Both organisms are sensitive to amikacin, gatifloxacin, ofloxacin, cefuroxime, gentamicin, ciprofloxacin, chloramphenicol, tobramycin, ceftazidime. 
The patient was commenced on a combination of ciprofloxacin $0.3 \%$ eye drop every half-hourly, fortified cefazolin $5 \%$ eyedrop every one-hourly and homatropine $2 \%$ eye drop three times daily. The patient was monitored regularly for next one week with same treatment [Figure 3- Figure 9]. After one week the infiltrate was completely resolved with scar formation. [Figure 10, Figure 11].

\section{Discussion:-}

Rigid gas permeable contact lenses provide sharper vision for irregular astigmatism, keratoconus, pellucid marginal corneal degeneration, post penetrating keratoplasty, keratoglobus and post-lasik ectasia patients. Prevalence of contact lens complications is more in soft contact lens wearers compare to rigid gas permeable wearers. ${ }^{5,6}$ The annual incidence of microbial keratitis in soft contact lenses wearers is 5.5 per 10,000, while 1.2 per 10,000 wearers of rigid contact lenses develop microbial keratitis ${ }^{7}$

Wearing contact lenses is one of the important risk factors for developing microbial keratitis, when using concomitantly with topical steroid eye drops, ocular trauma, ocular surgery and ocular surface disease. ${ }_{10,11,12,13,14}$ Microbial keratitis poses a major vision threat because considering that $80-90$ million people worldwide are wearing contact lenses for the correction of refractive errors. ${ }^{15}$ Moreover, the use of contact lenses may impair the immune-response of the cornea by distorting its epithelial-barrier function, contributing to the development of microbial keratitis. ${ }^{16}$ Contact lenses are a suitable surface for bacterial adhesion and biofilm formation. ${ }^{17}$ Microbial keratitis is as inflammation of cornea which characterized by excavation of the corneal epithelium, Bowman's layer, and stroma with infiltration and necrosis of tissue due to direct effects of infection by a microbial agent like bacteria, viruses, fungi or protozoa. ${ }^{18}$ Pseudomonas aeruginosa is the most common organism isolated of microbial keratitis in contact lens wearer. $5,6,7,11,1,2,19$.

Main symptoms of microbial keratitis are pain, redness, watering, foreign body sensation, mucous type discharge, vision disturbance, swollen eye lids but sometimes patients are asymptomatic. ${ }^{21}$ contact lens wearer has greater risk of microbial keratitis than that of non-contact lens wearer. ${ }^{10}$ Higher rates of complications are associated with men, with youth, with smokers, with longer periods of wear and with a lack of hand-washing. ${ }^{22,23}$ Pseudomonas keratitis typically occurs in contact lens wearer due to microbes enter into the eye at the time of insertion or removal of contact lens through finger, from lid margins, from the care solutionsorfromthestoragecase. ${ }^{17}$ Overnight lenses wear and sleeping with contact lenses are most significant factor for microbial keratitis. ${ }^{11,17}$ Orthokeratology is a process that uses specially designed rigid gas permeable contact lenses to temporarily reshape the contour of the cornea to reduce myopia, hyperopia, presbyopia. In Orthokeratology rigid gas-permeable contact lenses are normally worn only at night time, to improve vision through the reshaping of the cornea. There are very few cases reported in literature describing microbial keratitis associated with rigid gas permeable contact lenses in orthokeratology. ${ }^{24,25,26}$ Pseudomonas aeruginosa and acanthamoeba trophozoites organisms are more common pathogens involve in microbial keratitis related to Orthokeratology. ${ }^{24,25,26}$

Microbiological investigation should include contact lenses and contact lenses cleaning solutions, as this offer a clue for diagnosis and appropriate treatment of microbial keratitis. Microbial keratitis is an ocular emergency. Early diagnosis and effective management in the initial stage prevented from vision loss. Immediately contact lenses and contact lens solutions should be suspended. Anti- microbial therapy should be initiated immediately. Currently the "gold standard" of treatment for microbial keratitis has been a combination of two fortified antibiotic preparation(cefazolin 5\% and tobramycinl.3\%) which will cover whole range of grampositive and gram-negative pathogens or monotherapy with second generation fluoroquinolones (either ciprofloxacin $0.3 \%$ or ofloxacin $0.3 \%) .{ }^{27,28}$ Initially microbial keratitis should be monitored on daily basis. ${ }^{29}$ If pain, epithelial defects size and anterior chamber reaction decrease within 48 hours it may be considered to be effective treatment. ${ }^{30}$ If there is no sign of improvement within 48 hours then ciprofloxacin ointment at bed time may be useful. ${ }^{31}$ Also Mydriatics drugs are used to reduce pain from ciliary spasm , anterior chamber reaction and prevent the formation of posterior synechiae. ${ }^{27}$ If clinical condition did not improve with medications then therapeutic keratoplasty may be considered. ${ }^{30}$

\section{Conclusion:-}

Microbial keratitis is a serious and vision-threatening disease in which most of cases are associated with contact lens wear. Microbial keratitis is usually uncommon with rigid gas permeable contact lenses wearer. It can cause severe vision in loss in keratoconus patient. Corneal scraping should be done very carefully in keratoconus patient. Though the risk microbial keratitis is very low in rigid gas permeable contact lenses wearer than soft contact lenses wearer they still need optimum care and maintenance of contact lenses to reduce the contact lens related complications. 
Rigid Gas permeable (RGP) contact lens induced microbial keratitis in a keratoconus patient: A case

\begin{tabular}{|c|c|c|}
\hline \multicolumn{3}{|c|}{ Figure legends:- } \\
\hline $\begin{array}{l}\text { Figure 1 } \\
\text { Figure } 2 \\
\end{array}$ & $\begin{array}{l}\text { Picture of contact lenses } \\
\text { Culture. }\end{array}$ & \\
\hline $\begin{array}{l}\text { Figure } 3 \\
\text { Figure } 4 \text { (slit view) }\end{array}$ & $\begin{array}{l}1^{\text {st }} \text { day } \\
\text { Bi- microscopic appearance of a } \\
\text { contact lens related microbial } \\
\text { keratitis. }\end{array}$ & $\begin{array}{l}\text { Slit lamp picture obtained at } \\
\text { presentation showing corneal stromal } \\
\text { infiltration with dimensions } 2 \mathrm{~mm} \text { in } \\
\text { horizontally and } 1 \mathrm{~mm} \text { in vertically } \\
\text { with surrounding corneal oedema } \\
\text { and conjunctival injection. }\end{array}$ \\
\hline $\begin{array}{l}\text { Figure } 5 \\
\text { Figure } 6 \text { (slit view) }\end{array}$ & $2^{\text {nd }}$ day & $\begin{array}{l}\text { Showing reduction of corneal } \\
\text { Infiltration in size. }\end{array}$ \\
\hline $\begin{array}{l}\text { Figure } 7 \\
\text { Figure } 8 \text { (slit view) }\end{array}$ & $3^{\text {rd }}$ day follow up. & $\begin{array}{l}\text { Imaging obtained two days after first } \\
\text { presentation showing reduction of } \\
\text { corneal infiltration. }\end{array}$ \\
\hline Figure 9 & $4^{\text {th }}$ day follow up. & $\begin{array}{l}\text { Showing reduction of corneal } \\
\text { Infiltration in size and minimal } \\
\text { corneal stromal oedema present. }\end{array}$ \\
\hline Figure 10 & $8^{\text {th }}$ day follow up. & $\begin{array}{l}\text { The infiltration had healed } \\
\text { completely and resolved with small } \\
\text { circular scarring. }\end{array}$ \\
\hline Figure 11 & $8^{\text {th }}$ day follow up. & Slitlamp view of cornea in blue \\
\hline
\end{tabular}

\section{FIGURE:}

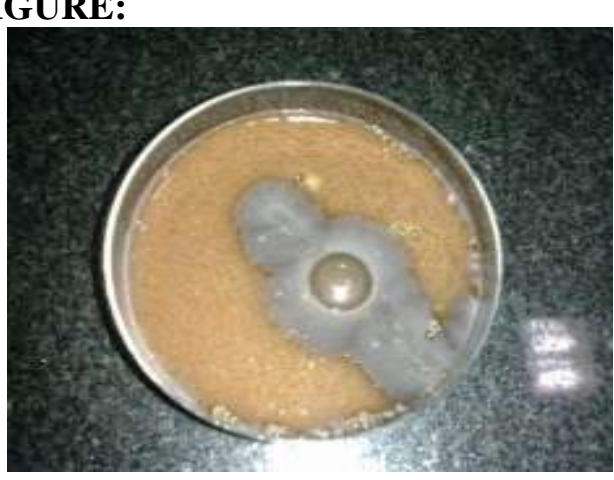

Figure 1
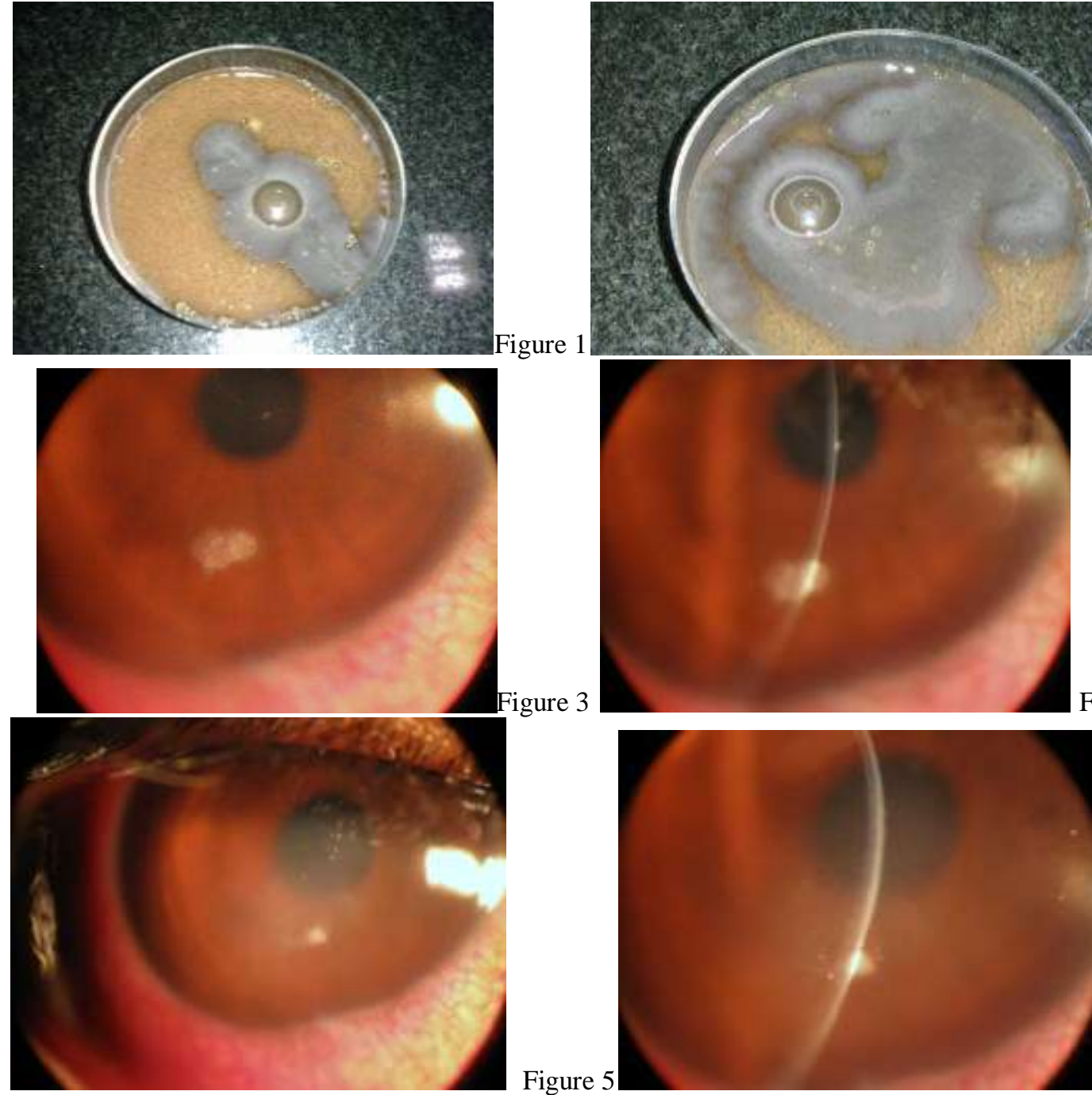

Figure 3

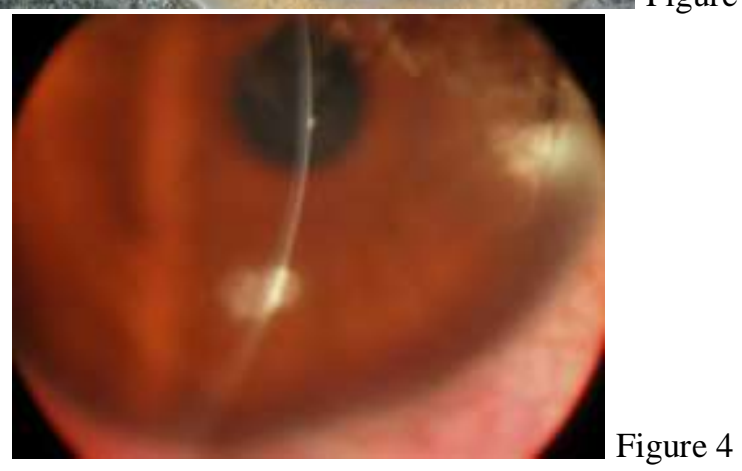

Figure 5

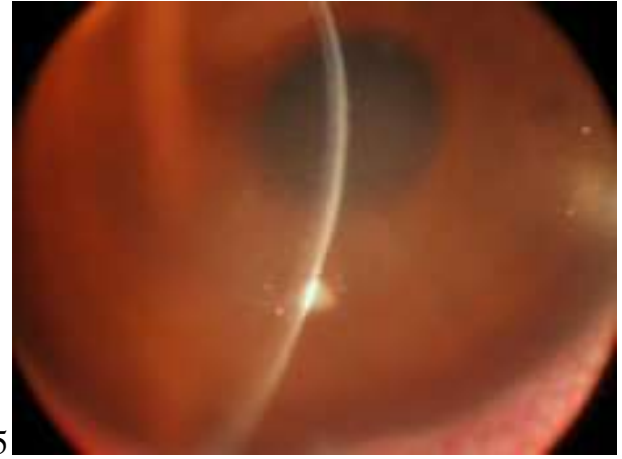

Figure 6 


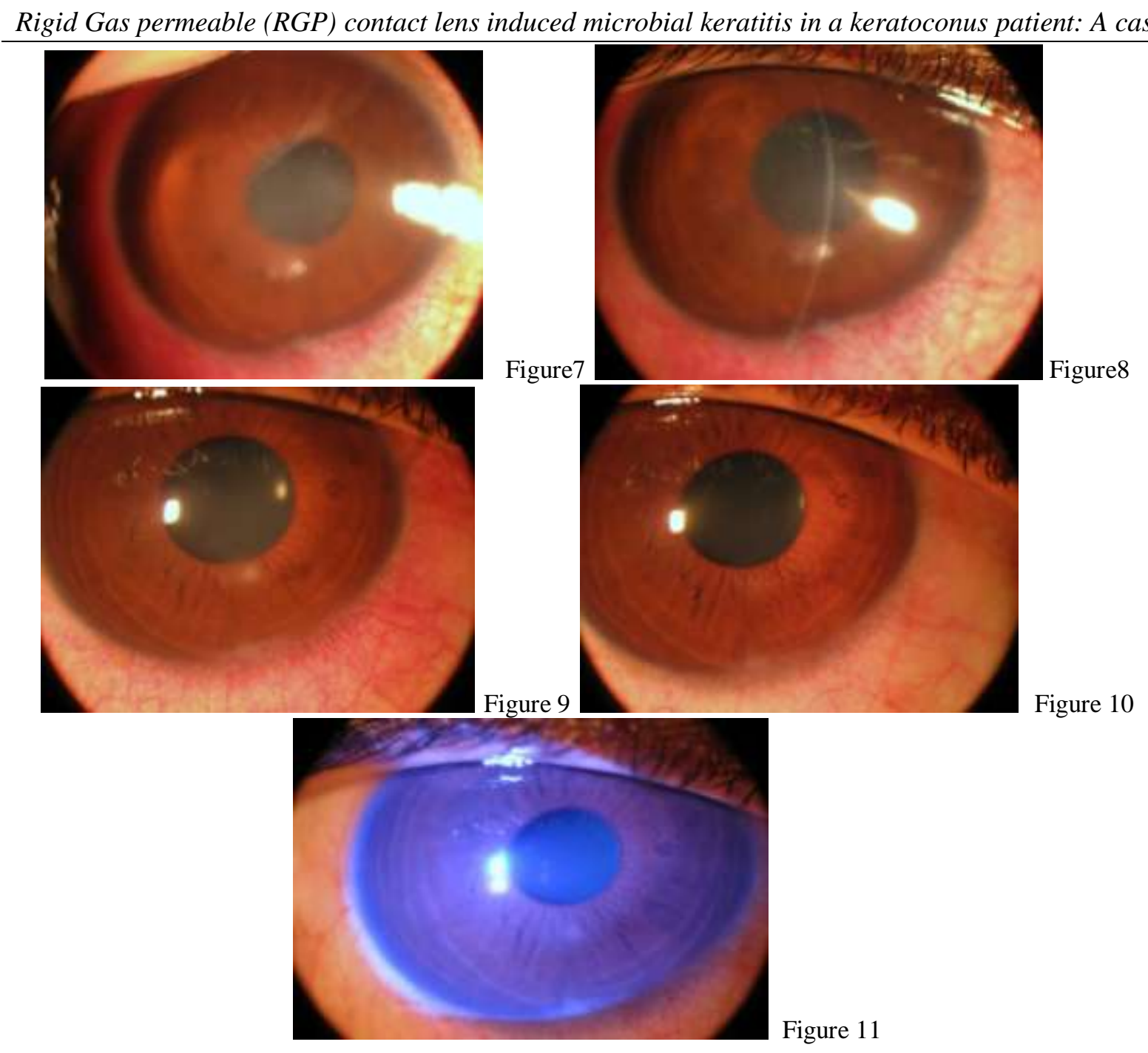

Acknowledgement:

I have taken efforts in this case report. However, it would not have been possible without the kind support and help of many individuals in my organizations. I would like to extend my sincere thanks to all of them. I would like to show my greatest appreciation to Mr. Anjul Kumar and Ms. Sana Quadri . I can't say thank you enough for his tremendous support and help. Without their encouragement and guidance this case report would not have materialized. The guidance and support received from all the members who contributed and who are contributing to this case report, was vital for the success of the case report. I am grateful for their constant support and help

\section{References:}

[1] Krachmer JH, Feder RS, Belin MW. Keratoconus and related noninflammatory corneal thinning disorders. Surv Ophthalmol. 1984;28: 293-322.

[2] Leibowitz HM, Morello S. Keratoconus and noninflammatory thinning disorders. In: Leibowitz HM, Waring GO, editors. Corneal disorders-clinical diagnosis and management. 2nd ed., Philadelphia, PA: WB Saunders Company; 1998. p. 349-74.

[3] Paulo Elias C. Dantas, M. Cristina Nishiwaki-Dantas. Spontaneous Bilateral Corneal Perforation of Acute Hydrops in Keratoconus. Eye \& Contact Lens.2004; 30:(l): 40-4l.

[4] Ladage PM, Yamamoto K, Ren DH, et al. Effects of rigid and soft contact lens daily wear on corneal epithelium, tear lactate dehydrogenase, and bacterial binding to exfoliated epithelial cells. Ophthalmology 2001;108: 1279-1288.

[5] Miller MJ, Wilson LA, Ahearn DG. Adherence of pseudomonas aeruginosa to rigid gas permeable contact lenses. Archives of Ophthalmology. 1991;109: 1447-1448.

[6] Nagachandrika T, Kumar T, et al. Prevalence of contact lens related complications in a tertiary eye centre in india. Contact lens\& Anterior eye. 2011Dec;34(6):266-8.

[7] Stapleton F, Keay L, Edwards K, et al. The incidence of contact lens-related microbial keratitis in Australia. Ophthalmology 2008; 115:1655-1662.

[8] Ching-His Hsiao, Ling Yeung, et al. Pediatric Microbial Keratitis in Taiwanese Children. Arch Ophthalmol. 2007; 125:603-609.

[9] Richard G Lindsay, Richard Johnson, et al. Acanthamoeba keratitis and contact lens wear. Clin Exp Optom 2007; 90: 5: 351-360.

[10] Bennie H. Jeng, Abha B. kumar, et, al. Epidemiology of Ulcerative Keratitis in Northern California. Arch Ophthalmol. $2010 . ; 128$ ( 8):1022-1028. 
[11] Al-Yousuf N.Microbial keratitis in kingdom of Bahrain: clinical and microbiology study. Middle East Afr J Ophthalmol. 2009;16:3-7.

[12] Fleiszig SM, The GlennA.Fry award lecture 2005. The Pathogenesis of contact lens-related keratitis. Optom Vis Sci. 2006;83:866-73.

[13] Poggio EC, Glynn RJ, Schein OD, et al. The incidence of ulcerative keratitis among users of daily-wear and extended-wear soft contact lenses. N Engl J Med 1989;321:779-783.

[14] Erie JC, Nevitt MP, Hodge DO, Ballard DJ. Incidence of ulcerative keratitis in a defined population from 1950 through 1988. Arch Ophthalmol 1993;111:1665-1671.

[15] Ren DH, Yamamoto K, Ladage PM, et al. Adaptive effects of 30-night wear of hyper-O(2) transmissible contact lenses on bacterial binding and corneal epithelium: a 1- year clinical trial. Ophthalmology 2002;109:27-39; discussion 39-40.

[16] Liesegang TJ. Physiologic changes of the cornea with contact lens wear. CLAO J 2002;28:12-27.

[17] Fleiszig SM, Evans DJ. Pathogenesis of contact lens-associated microbial keratitis. Optom Vis Sci. 2010;87:225-232.

[18] Cokington CD, Hyndiuk RA. Bacterial keratitis. In Infection of the Eye. Second Edition. Little, Brown \& Co, USA, 1996.

[19] K. Tabbara, H. El-Sheikh, and B. Aabed. Extended wear contact lens related bacterial keratitis: Br J Ophthalmol. 2000; 84(3): $327-328$.

[20] Carmichael TR, Wolpert M, Koornhof HJ. Corneal ulceration at an urban African hospital. Br J Ophthalmol 1985; 69:920-6.

[21] Keay, Lisa, Edwards, Katie†; Stapleton, Fiona .Signs, Symptoms, and Comorbidities in Contact Lens-Related Microbial Keratitis. Optometry \& Vision Science: 2009: 86: 803-809.

[22] Wu YT, Zhu H, Harmis NY, Iskandar SY, Willcox M, Stapleton F. Profile and frequency of microbial contamination of contact lens cases. Optom Vis Sci. 2010;87:153-158.

[23] Yeung KK, Forister JF, Forister EF, Chung MY, Han S, Weissman BA. Compliance with soft contact lens replacement schedules and associated contact lens-related ocular complications: The UCLA Contact Lens Study. Optometry. 2010;81:598-607.

[24] Wilhelmus, Kirk R MD, PhD. Acanthamoeba Keratitis During Orthokeratology. Cornea: 2005; 24(7): 864-866.

[25] Ying-Cheng, Lin M.D.; Chao-Kung, Lu M.D.; Ko-Hua, et ai. Daytime Orthokeratology Associated With Infectious Keratitis by Multiple Gram-Negative Bacilli: Burkholderia cepacia, Pseudomonas putida, and Pseudomonas aeruginosa. Eye \& Contact Lens:2006 ;32(1):19-20.

[26] Shehadeh-Masha'our R, Segev F, Barequet IS, Ton Y, Garzozi H J. Orthokeratology associated microbial keratitis. Eur J Ophthalmol 2009;19:133-6.

[27] Kanski JJ. Clinical ophthalmology: a synopsis. 2nd ed. Oxford: Butterworth-Heinemann; 2009. p. 138-150.

[28] Chawla B, Agarwal P, Tandon R, et al. In vitro susceptibility of bacterial keratitis isolates to fourth-generation fluoroquinolones. Eur J Ophthalmol. 2010;20:300-305.

[29] Friedman NJ, Kaiser PK, Pineda R. The Massachusetts eye and ear infirmary illustrated manual of ophthalmology. 3rd ed. Philadelphia: Elsevier; 2009. p. 188-204.

[30] Ehlers JP, Shah CP. The Wills eye manual: office and emergency room diagnosis and treatment of eye disease. 5th Ed. Baltimore: Lippincott Williams \& Wilkins; 2008. p. 62-86. 